Pacific

Journal of

Mathematics

HEISENBERG UNCERTAINTY PRINCIPLE ON CHÉBLI-TRIMÈCHE HYPERGROUPS

RUIQIN MA 


\title{
HEISENBERG UNCERTAINTY PRINCIPLE ON CHÉBLI-TRIMÈCHE HYPERGROUPS
}

\author{
RUIQIN MA
}

\begin{abstract}
An uncertainty principle on Chébli-Trimèche hypergroups is established, as a generalization of Heisenberg inequalities for Jacobi transforms proved in my previous paper. It implies and extends the uncertainty principle for Hankel transforms by M. Rösler and M. Voit. The proof is based on ultracontractive properties of the semigroups generated by a second order differential operator and on the estimate of the heat kernel.
\end{abstract}

\section{Introduction}

The classical Heisenberg uncertainty principle states that for $f \in \mathrm{L}^{2}(\mathbb{R})$,

$$
\int_{\mathbb{R}} x^{2}|f(x)|^{2} d x \cdot \int_{\mathbb{R}} \xi^{2}|\widehat{f}(\xi)|^{2} d \xi \geq \frac{1}{4}\|f\|^{4},
$$

where

$$
\widehat{f}(\xi)=\frac{1}{\sqrt{2 \pi}} \int_{\mathbb{R}} f(x) e^{-i \xi x} d x .
$$

Inequality (1-1) was found in the 1920's and then studied in many situations. Recently, considerable attention has been devoted to discovering new contexts for the uncertainty principle; see the survey [Folland and Sitaram 1997] and the book [Havin and Jöricke 1994] for other forms of the uncertainty principle. In the case of hypergroups, the Heisenberg inequalities were given in [Li and Liu 2005] for some compact Chébli-Trimèche hypergroups, in [Rösler and Voit 1999] for Bessel-Kingman hypergroups, and in [Ma 2007] for Jacobi hypergroups.

This paper generalizes the results of my previous paper [Ma 2007] to noncompact Chébli-Trimèche hypergroups and establishes an inequality analogous to (1-1) and variants of it. The proof is based on ultracontractive properties of the semigroups generated by a second order differential operator and on the estimate of the heat kernel. For further uncertainty principles on Chébli-Trimèche hypergroups, see [Attour and Trimèche 2005; Bouattour and Trimèche 2005; Trimèche 2005].

MSC2000: primary 43A62; secondary 44A15.

Keywords: uncertainty principle, hypergroups.

This work was supported by the Foundation of Beijing Institute of Technology. 
The paper is arranged as follows. In Section 2, we recall some basic properties about Chébli-Trimèche hypergroups. In Section 3, we study the Heisenberg uncertainty principle on them.

\section{Preliminaries of Chébli-Trimèche hypergroups}

We now recall some definitions and properties of Chébli-Trimèche hypergroups; see also [Bloom and Xu 1995; Trimèche 1997].

Suppose that the function $A$ is continuous on $\mathbb{R}_{+}=[0, \infty)$, twice continuously differentiable on $\mathbb{R}_{+}^{*}=(0, \infty)$, and satisfies the conditions:

(1) $A(0)=0$ and $A(x)>0$ for $x>0$;

(2) $A$ is increasing and unbounded;

(3) $A^{\prime}(x) / A(x)=(2 \alpha+1) / x+B(x)$ on a neighborhood of 0 , where $\alpha>-1 / 2$ and $B$ is an odd $C^{\infty}$-function on $\mathbb{R}$;

(4) $A^{\prime}(x) / A(x)$ is a decreasing $C^{\infty}$-function on $\mathbb{R}_{+}^{*}$, and $\lim _{x \rightarrow+\infty} A^{\prime}(x) / A(x)=$ $2 \rho \geq 0$.

We denote by $\left(\mathbb{R}_{+}, *(A)\right)$ the Chébli-Trimèche hypergroup associated with $A$. It is commutative with neutral element 0 and the identity mapping is the involution. The Haar measure on $\left(\mathbb{R}_{+}, *(A)\right)$ is given by $A(x) d x$.

Well-known Chébli-Trimèche hypergroups are the Bessel-Kingman hypergroup with $A(x)=x^{2 \alpha+1}$ for $\alpha>-1 / 2$ and the Jacobi hypergroup defined by $A(x)=$ $\sinh ^{2 \alpha+1} x \cosh ^{2 \beta+1} x$, where $\alpha \geq \beta \geq-1 / 2$ and $\alpha \neq-1 / 2$.

Let $\mathscr{L}=\mathscr{L}_{A}$ be the differential operator

$$
\mathscr{L}=\frac{d^{2}}{d x^{2}}+\left(\frac{A^{\prime}(x)}{A(x)}\right) \frac{d}{d x} .
$$

The solutions $\phi_{\lambda}$ for $\lambda \in \mathbb{C}$ of the differential equation $\mathscr{L} v(x)=\left(\lambda^{2}+\rho^{2}\right) v(x)$ with boundary conditions $v(0)=1$ and $v^{\prime}(0)=0$ are multiplicative on $\left(\mathbb{R}_{+}, *(A)\right)$ in that, for all $x, y \in \mathbb{R}_{+}^{*}$,

$$
\int_{\mathbb{R}_{+}} \phi_{\lambda}(t)\left(\varepsilon_{x} * \varepsilon_{y}\right)(d t)=\phi_{\lambda}(x) \phi_{\lambda}(y),
$$

where $\varepsilon_{x}$ denotes the point mass at $x$ and $\varepsilon_{x} * \varepsilon_{y}$ is a probability measure that is absolutely continuous with respect to the Haar measure and satisfies

$$
\operatorname{supp} \varepsilon_{x} * \varepsilon_{y} \subset[|x-y|, x+y] \text {. }
$$

The dual space of $\left(\mathbb{R}_{+}, *(A)\right)$ can be identified with the parameter set $\mathbb{R}_{+} \cup i[0, \rho]$. 
For $1 \leq p \leq \infty$, the Lebesgue space $L^{p}\left(\mathbb{R}_{+}, A(x) d x\right)$ is defined as usual with norm given by $\|\cdot\|_{p}$. The Fourier transform of $f$ in $L^{1}\left(\mathbb{R}_{+}, A(x) d x\right)$ is given by

$$
\widehat{f}(\lambda):=\int_{0}^{\infty} f(x) \phi_{\lambda}(x) A(x) d x .
$$

Theorem 2.1 [Bloom and Heyer 1995]. There exists a unique nonnegative measure $\pi$ on $\mathbb{R}_{+}$such that the Fourier transform extends to an isometry of $L^{2}\left(\mathbb{R}_{+}, A(x) d x\right)$ onto $L^{2}\left(\mathbb{R}_{+}, \pi\right)$, and for $f \in L^{1} \cap L^{2}\left(\mathbb{R}_{+}, A(x) d x\right)$,

$$
\int_{0}^{\infty}|f(x)|^{2} A(x) d x=\int_{0}^{\infty}|\widehat{f}(\lambda)|^{2} \pi(d \lambda) .
$$

As in [Bloom and $\mathrm{Xu} 1995]$, here we assume that $A$ satisfies an additional property, which gives a nice behavior for the Plancherel measure $\pi$.

Condition 2.1. A function $f$ is said to satisfy this condition if, for some $\mu>0$ and $x_{0}>0$,

$$
\int_{x_{0}}^{\infty} x^{\varpi(\mu)}|\zeta(x)| d x<\infty, \quad \text { where } \zeta(x)=f(x)-\frac{\mu^{2}-1 / 4}{x^{2}},
$$

and if $\zeta(x)$ is bounded for $x>x_{0}$; here $\varpi(\mu)=\mu+1 / 2$ if $\mu \geq 1 / 2$ and $\varpi(\mu)=1$ otherwise.

Letting

$$
G(x)=\frac{1}{4}\left(\frac{A^{\prime}(x)}{A(x)}\right)^{2}+\frac{1}{2}\left(\frac{A^{\prime}(x)}{A(x)}\right)^{\prime}-\rho^{2} \quad \text { for } x>0,
$$

we have the following theorem from [Bloom and Xu 1995].

Theorem 2.2. Suppose that $G$ satisfies Condition 2.1 together with one of the conditions

(1) $\mu>1 / 2$;

(2) $\mu \neq|\alpha|$;

(3) $\mu=\alpha \leq \frac{1}{2}$ and either $\int_{0}^{\infty} x^{1 / 2-\alpha} \zeta(x) \phi_{0}(x) A(x)^{1 / 2} d x \neq-2 \alpha \sqrt{M_{A}}$, or

$$
\int_{0}^{\infty} x^{1 / 2+\alpha} \zeta(x) \phi_{0}(x) A(x)^{1 / 2} d x=0,
$$

where $M_{A}=\lim _{x \rightarrow 0^{+}} x^{-2 \alpha-1} A(x)$.

Then the Plancherel measure $\pi$ is absolutely continuous in the Lebesgue measure and has density $|c(\lambda)|^{-2}$, where the function $c$ is such that there exist positive constants $C_{1}, C_{2}$, and $K$ such that, for any $\lambda \in \mathbb{C}$ with $\operatorname{Im} \lambda \leq 0$,

$$
\begin{array}{ll}
C_{1}|\lambda|^{\mu+1 / 2} \leq|c(\lambda)|^{-1} \leq C_{2}|\lambda|^{\mu+1 / 2} & \text { for }|\lambda| \leq K, \\
C_{1}|\lambda|^{\alpha+1 / 2} \leq|c(\lambda)|^{-1} \leq C_{2}|\lambda|^{\alpha+1 / 2} & \text { for }|\lambda|>K .
\end{array}
$$


For $1 \leq p \leq+\infty$, denote by $L^{p}\left(\mathbb{R}_{+}, d v\right)$ the space of measurable functions $f$ on $[0, \infty)$ such that

$$
\begin{aligned}
\|f\|_{p, c} & =\left(\int_{0}^{\infty}|f(\lambda)|^{p}|c(\lambda)|^{-2} d \lambda\right)^{1 / p}<\infty, & 1 \leq p<\infty, \\
\|f\|_{\infty}=\operatorname{ess} \sup _{\lambda \geq 0}|f(\lambda)|<\infty, & p & =\infty,
\end{aligned}
$$

The generalized translate $T_{x}$ of a function $f$ is defined by

$$
T_{x} f(y)=\int_{0}^{\infty} f(u)\left(\varepsilon_{x} * \varepsilon_{y}\right)(d u) .
$$

The convolution of two functions $f$ and $g$ is defined by

$$
f * g(x)=\int_{0}^{\infty} T_{x} f(y) g(y) A(y) d y .
$$

The convolution satisfies the following properties (see [Trimèche 1997]).

(1) For all $f, g \in L^{1}\left(\mathbb{R}_{+}, A(x) d x\right)$, the function $f * g$ is defined almost everywhere on $\mathbb{R}_{+}$, belongs to $L^{1}\left(\mathbb{R}_{+}, A(x) d x\right)$, and satisfies

$$
\widehat{f * g}(\lambda)=\widehat{f}(\lambda) \widehat{g}(\lambda) \text {. }
$$

(2) Let $f$ and $g$ be in $L^{2}\left(\mathbb{R}_{+}, A(x) d x\right)$. Then the function $f * g$ belongs to $L^{2}\left(\mathbb{R}_{+}, A(x) d x\right)$ if and only if the function $\hat{f} \cdot \hat{g}$ belongs to $L^{2}\left(\mathbb{R}_{+}, d v\right)$ and

$$
\widehat{f * g}(\lambda)=\widehat{f}(\lambda) \widehat{g}(\lambda) .
$$

(3) Suppose $f \in L^{p}\left(\mathbb{R}_{+}, A(x) d x\right)$ and $g \in L^{q}\left(\mathbb{R}_{+}, A(x) d x\right)$ with $1 \leq p, q, r \leq \infty$ and $1 / p+1 / q-1=1 / r$. Then $f * g \in L^{r}\left(\mathbb{R}_{+}, A(x) d x\right)$ and

$$
\|f * g\|_{r} \leq\|f\|_{p}\|g\|_{q} .
$$

\section{Uncertainty principle on Chébli-Trimèche hypergroups}

In the sequel, we assume that $A$ satisfies the Condition 2.1 in Theorem 2.2. In addition, we also suppose that in the case $\rho=0$, there exists a $\beta \in(-1 / 2, \mu]$ such that $A(x)=O\left(x^{2 \beta+1}\right)$ as $x \rightarrow \infty$.

Let $D_{\rho}:=[(\beta+1) /(\mu+1), 1]$ if $\rho=0$. If $0<\rho<1$, let $D_{\rho}:=(1 / 2,1]$; otherwise let $D_{\rho}:=[1 / 2,1]$.

Theorem 3.1. Assume $a, b>0$ and $\gamma \in D_{\rho}$. Then there exists a constant $C>0$ such that

$$
\left\|x^{\gamma a} f\right\|_{2}^{b /(a+b)} \cdot\left\|\left(\lambda^{2}+\rho^{2}\right)^{b / 2} \widehat{f}\right\|_{2, c}^{a /(a+b)} \geq C\|f\|_{2},
$$

for all $f \in L^{2}\left(\mathbb{R}_{+}, A(x) d x\right)$. 
Remarks. (1) For Bessel-Kingman hypergroups, $\rho=0$ and $\beta=\mu=\alpha$, So (3-1) becomes

$$
\left\|x^{a} f\right\|_{2}^{b /(a+b)} \cdot\left\|\lambda^{b} \widehat{f}\right\|_{2, c}^{a /(a+b)} \geq C\|f\|_{2},
$$

which extends the result in [Rösler and Voit 1999].

(2) In the case of Jacobi hypergroups, $\rho>0$ and $\mu=1 / 2$. It's just the uncertainty principle for Jacobi transform, which improves the original theorem in [Ma 2007].

(3) If there exists a $\delta>0$ such that for all $x \in\left[x_{0}, \infty\right.$ ) ( for some $x_{0}>0$ ),

$$
\frac{A^{\prime}(x)}{A(x)}= \begin{cases}2 \rho+e^{-\delta x} D(x), & \text { if } \rho>0, \\ (2 \alpha+1) / x+e^{-\delta x} D(x) & \text { if } \rho=0,\end{cases}
$$

where $D$ and its derivatives are bounded $C^{\infty}$ functions, we have $\mu=1 / 2$ if $\rho>0$, and $\beta=\mu=\alpha$ if $\rho=0$ and $\alpha>0$; see [Trimèche 1997].

Lemma 3.1 [Bloom and Xu 1995]. $A(x) \sim x^{2 \alpha+1}$ as $x \rightarrow 0^{+}$, and if $\rho>0$ then $A(x) \sim e^{2 \rho x}$ as $x \rightarrow+\infty$. Here $f \sim g$ means that there exist positive constants $C_{1}$ and $C_{2}$ such that $C_{1} g \leq f \leq C_{2} g$.

In our proof, the heat kernel $h_{t}(x)$, given by

$$
h_{t}(x)=\int_{0}^{\infty} e^{-t\left(\lambda^{2}+\rho^{2}\right)} \phi_{\lambda}(x)|c(\lambda)|^{-2} d \lambda \quad \text { for } t>0
$$

plays an important role. For all $t>0$ and $f \in L^{2}\left(\mathbb{R}_{+}, A(x) d x\right)$, we have

$$
\widehat{h_{t} * f}(\lambda)=e^{-t\left(\lambda^{2}+\rho^{2}\right)} \hat{f}(\lambda) \text {. }
$$

We can now prove nice estimates for $\left\|h_{t}\right\|_{2}$.

\section{Lemma 3.2.}

$$
\left\|h_{t}\right\|_{2} \sim \begin{cases}t^{-(\alpha+1) / 2} & \text { if } t \leq 1 \\ e^{-t \rho^{2}} t^{-(\mu+1) / 2} & \text { if } t>1\end{cases}
$$

Proof.

$$
\left\|h_{t}\right\|_{2}=\left(\int_{0}^{\infty} e^{-2 t\left(\lambda^{2}+\rho^{2}\right)}|c(\lambda)|^{-2} d \lambda\right)^{1 / 2}=e^{-t \rho^{2}}\left(\int_{0}^{\infty} e^{-2 t \lambda^{2}}|c(\lambda)|^{-2} d \lambda\right)^{1 / 2} .
$$

Using the estimates of the $c$-function,

$$
|c(\lambda)|^{-2} \sim \begin{cases}\lambda^{2 \mu+1} & \text { if } t \leq K, \\ \lambda^{2 \alpha+1} & \text { if } t>K .\end{cases}
$$

Then

$$
\begin{aligned}
\left\|h_{t}\right\|_{2} & \sim e^{-t \rho^{2}}\left(\int_{0}^{K} e^{-2 t \lambda^{2}} \lambda^{2 \mu+1} d \lambda+\int_{K}^{\infty} e^{-2 t \lambda^{2}} \lambda^{2 \alpha+1} d \lambda\right)^{1 / 2} \\
& \sim e^{-t \rho^{2}}\left(t^{-(\mu+1)} \int_{0}^{2 t K^{2}} e^{-s} s^{\mu} d s+t^{-(\alpha+1)} \int_{2 t K^{2}}^{\infty} e^{-s} s^{\alpha} d s\right)^{1 / 2}
\end{aligned}
$$


where the second equivalence is obtained by a change of variables. Because $t \sim 0$, the first term in curly braces is bounded, and the second is equivalent to $t^{-(\alpha+1)}$. When $t$ tends to infinity, the first term is equivalent to $t^{-(\mu+1)}$. By the relation between the incomplete gamma function $\Gamma(\cdot, \cdot)$ and the confluent hypergeometric function $G(\cdot, \cdot, \cdot)$ (see [Nikiforov and Uvarov 1988, pages 401-413]), we know that the second term equals

$$
\begin{aligned}
t^{-(\alpha+1)} \Gamma\left(\alpha+1,2 t K^{2}\right) & =t^{-(\alpha+1)} e^{-2 t K^{2}}\left(2 t K^{2}\right)^{\alpha+1} G\left(1,2+\alpha, 2 t K^{2}\right) \\
& \sim e^{-2 t K^{2}} G\left(1,2+\alpha, 2 t K^{2}\right) \sim e^{-2 t K^{2}} t^{-1} \quad \text { as } t \rightarrow \infty
\end{aligned}
$$

The lemma is proved by the above equivalences.

The constant $C$ below is not fixed and might change appropriately in different equalities or inequalities.

Lemma 3.3. Assume $\gamma \in D_{\rho}$. Then for all $f \in L^{2}\left(\mathbb{R}_{+}, A(x) d x\right)$ we have

$$
\left\|h_{t} * f\right\|_{2} \leq C t^{-a / 2}\left\|x^{\gamma a} f\right\|_{2},
$$

where $a>0$ and satisfies $\gamma a<\alpha+1$ if $\rho>0$ and $\gamma a<\min \{\alpha+1, \beta+1\}$ otherwise.

Proof. We only give the proof for $\rho>0$; the case $\rho=0$ can be got similarly. For $r>0$, let $f_{r}=f \chi_{[0, r]}$ and $f^{r}=f-f_{r}$. Then, since $\left|f^{r}(x)\right| \leq r^{-\gamma a}\left|x^{\gamma a} f(x)\right|$,

$$
\left\|h_{t} * f^{r}\right\|_{2}=\left\|e^{-t\left(\lambda^{2}+\rho^{2}\right)} \widehat{f^{r}}(\lambda)\right\|_{2, c} \leq\left\|\widehat{f^{r}}\right\|_{2, c}=\left\|f^{r}\right\|_{2} \leq r^{-\gamma a}\left\|x^{\gamma a} f\right\|_{2} .
$$

On the other hand, we have

$$
\left\|f_{r} * h_{t}\right\|_{2} \leq\left\|f_{r}\right\|_{1}\left\|h_{t}\right\|_{2} \leq\left\|h_{t}\right\|_{2}\left(\int_{0}^{r} x^{-2 \gamma a} A(x) d x\right)^{1 / 2}\left\|x^{\gamma a} f\right\|_{2} .
$$

By Lemma 3.1, $A(x) \sim e^{2 \rho x}$ when $x$ is big, and $A(x) \sim x^{2 \alpha+1}$ when $x \sim 0$. Under the assumption on $\gamma$, we have that there exists some positive constant $d$ such that

$$
\left(\int_{0}^{r} x^{-2 \gamma a} A(x) d x\right)^{1 / 2} \leq d r^{-\gamma a} V(r), \quad \text { where } \quad V(r)= \begin{cases}r^{\alpha+1} & \text { if } r \leq 1 \\ r^{1 / 2} e^{\rho r} & \text { if } r>1 .\end{cases}
$$

So

$$
\begin{aligned}
\left\|h_{t} * f\right\|_{2} & \leq\left\|h_{t} * f_{r}\right\|_{2}+\left\|h_{t} * f^{r}\right\|_{2} \\
& \leq C r^{-\gamma a}\left(1+\left\|h_{t}\right\|_{2} V(r)\right)\left\|x^{\gamma a} f\right\|_{2} .
\end{aligned}
$$

Choosing $r=t^{1 /(2 \gamma)}$ for $\gamma \in D_{\rho}$, we obtain (3-2) by Lemma 3.2.

Proof of Theorem 3.1. We only prove the case $\rho>0$, as it can be proved similarly for $\rho=0$. Assume that $\gamma \in D_{\rho}$ for $\gamma a<\alpha+1$. If $b \leq 2$, it suffices to prove that

$$
\left\|x^{\gamma a} f\right\|_{2}^{b /(a+b)} \cdot\left\|\left(\lambda^{2}+\rho^{2}\right)^{b / 2} \widehat{f}(\lambda)\right\|_{2, c}^{a /(a+b)} \geq C\|f\|_{2} .
$$

for $f$ and $x^{\gamma a} f$ in $L^{2}\left(R_{+}, A(x) d x\right)$ and $\left(\lambda^{2}+\rho^{2}\right)^{b / 2} \widehat{f}(\lambda)$ in $L^{2}\left(R_{+}, d \nu\right)$. 
By (3-2),

$$
\begin{aligned}
& \|f\|_{2} \leq\left\|h_{t} * f\right\|_{2}+\left\|f-h_{t} * f\right\|_{2} \\
& \quad \leq C t^{-a / 2}\left\|x^{\gamma a} f\right\|_{2}+\left\|\left(1-e^{-t\left(\lambda^{2}+\rho^{2}\right)}\right) \hat{f}(\lambda)\right\|_{2, c} \\
& \quad=C t^{-a / 2}\left\|x^{\gamma a} f\right\|_{2}+\left\|\left(1-e^{-t\left(\lambda^{2}+\rho^{2}\right)}\right)\left(t\left(\lambda^{2}+\rho^{2}\right)\right)^{-b / 2}\left(t\left(\lambda^{2}+\rho^{2}\right)\right)^{b / 2} \hat{f}(\lambda)\right\|_{2, c} .
\end{aligned}
$$

The size of last term is controlled by $t^{b / 2}\left\|\left(\lambda^{2}+\rho^{2}\right)^{b / 2} \hat{f}(\lambda)\right\|_{2, c}$, since the function $\left(1-e^{-s}\right) s^{-b / 2}$ is bounded for $s \geq 0$ if $b \leq 2$. Therefore

$$
\|f\|_{2} \leq C\left(t^{-a / 2}\left\|x^{\gamma a} f\right\|_{2}+t^{b / 2}\left\|\left(\lambda^{2}+\rho^{2}\right)^{b / 2} \hat{f}(\lambda)\right\|_{2, c}\right),
$$

from which, optimizing in $t$, we obtain (3-1) for $\gamma a<\alpha+1$ and $b \leq 2$.

If $b>2$, let $b^{\prime} \leq 2$. For $u \geq 0$ and $b^{\prime}<b$, we have $u^{b^{\prime}} \leq 1+u^{b}$ which, for $u=\left(\left(\lambda^{2}+\rho^{2}\right) / \epsilon\right)^{1 / 2}$ gives the inequality $\left(\left(\lambda^{2}+\rho^{2}\right) / \epsilon\right)^{b^{\prime} / 2} \leq 1+\left(\left(\lambda^{2}+\rho^{2}\right) / \epsilon\right)^{b / 2}$ for all $\epsilon>0$.

It follows that

$$
\left\|\left(\lambda^{2}+\rho^{2}\right)^{b^{\prime} / 2} \widehat{f}\right\|_{2, c} \leq \epsilon^{b^{\prime} / 2}\|f\|_{2}+\epsilon^{\left(b^{\prime}-b\right) / 2}\left\|\left(\lambda^{2}+\rho^{2}\right)^{b / 2} \widehat{f}\right\|_{2, c} .
$$

Optimizing in $\epsilon$, we get

$$
\left\|\left(\lambda^{2}+\rho^{2}\right)^{b^{\prime} / 2} \widehat{f}\right\|_{2, c} \leq\|f\|_{2}^{1-b^{\prime} / b}\left\|\left(\lambda^{2}+\rho^{2}\right)^{b / 2} \widehat{f}\right\|_{2, c}^{b^{\prime} / b} .
$$

Together with (3-1) for $b^{\prime}$, we get the result for $b>2$.

If $\gamma a \geq \alpha+1$, let $\gamma a^{\prime}<\alpha+1$. Then using

$$
\frac{x^{\gamma a^{\prime}}}{\epsilon^{\gamma a^{\prime}}} \leq 1+\frac{x^{\gamma a}}{\epsilon^{\gamma a}} \quad \text { for } \epsilon>0
$$

we get the result similarly.

\section{Acknowledgment}

The author thanks the referee for many valuable comments and suggestions.

\section{References}

[Attour and Trimèche 2005] L. B. Attour and K. Trimèche, "Uncertainty principle and $\left(L^{p}, L^{q}\right)$ sufficient pairs on Chébli-Trimèche hypergroups", Integral Transforms Spec. Funct. 16:8 (2005), 625-637. MR 2007c:43005 Zbl 1089.43004

[Bloom and Heyer 1995] W. R. Bloom and H. Heyer, Harmonic analysis of probability measures on hypergroups, de Gruyter Studies in Mathematics 20, Walter de Gruyter, Berlin, 1995. MR 96a: 43001 Zbl 0828.43005

[Bloom and Xu 1995] W. R. Bloom and Z. F. Xu, "The Hardy-Littlewood maximal function for Chébli-Trimèche hypergroups", pp. 45-70 in Applications of hypergroups and related measure algebras (Seattle, WA, 1993), edited by W. C. Connett et al., Contemp. Math. 183, Amer. Math. Soc., Providence, RI, 1995. MR 96c:43013 Zbl 0834.42011 
[Bouattour and Trimèche 2005] L. Bouattour and K. Trimèche, "Beurling-Hörmander's theorem for the Chébli-Trimèche transform”, Glob. J. Pure Appl. Math. 1:3 (2005), 342-357. MR 2007h:43002 Zbl 1105.43004

[Folland and Sitaram 1997] G. B. Folland and A. Sitaram, "The uncertainty principle: a mathematical survey”, J. Fourier Anal. Appl. 3:3 (1997), 207-238. MR 98f:42006 Zbl 0885.42006

[Havin and Jöricke 1994] V. Havin and B. Jöricke, The uncertainty principle in harmonic analysis, Ergebnisse der Mathematik (3) 28, Springer, Berlin, 1994. MR 96c:42001 Zbl 0827.42001

[Li and Liu 2005] Z. Li and L. Liu, "Uncertainty principles for Sturm-Liouville operators", Constr. Approx. 21:2 (2005), 193-205. MR 2005h:34219 Zbl 1072.34095

[Ma 2007] R. Ma, "Heisenberg inequalities for Jacobi transforms", J. Math. Anal. Appl. 332:1 (2007), 155-163. MR 2319650 Zbl 1116.43008

[Nikiforov and Uvarov 1988] A. F. Nikiforov and V. B. Uvarov, Special functions of mathematical physics, Birkhäuser, Basel, 1988. MR 89h:33001 Zbl 0624.33001

[Rösler and Voit 1999] M. Rösler and M. Voit, "An uncertainty principle for Hankel transforms", Proc. Amer. Math. Soc. 127:1 (1999), 183-194. MR 99i:44007 Zbl 0910.44003

[Trimèche 1997] K. Trimèche, Generalized wavelets and hypergroups, Gordon and Breach Science Publishers, Amsterdam, 1997. MR 99h:43014 Zbl 0926.42016

[Trimèche 2005] K. Trimèche, "Cowling-Price and Hardy theorems on Chébli-Trimèche hypergroups", Glob. J. Pure Appl. Math. 1:3 (2005), 286-305. MR 2007f:43004 Zbl 1122.43005

Received August 20, 2007. Revised December 5, 2007.

\author{
RUIQIN MA \\ SCHOOL OF SCIENCES \\ BEIJING InSTITUTE OF TECHNOLOGY \\ BEIJING 100081 \\ CHINA \\ rqma@163.com
}

